\title{
A review on positioning techniques and technologies: a novel AI approach
}

\begin{abstract}
There are variety of positioning techniques applied to tracking of mobile objects such as mobile robots, handheld devices carried by human subject, etc. With the advent of new technologies, new strategies have emerged from combination of algorithms and those technical capabilities. This study is dedicated to a review of past and current approaches to positioning, including their advantages and shortcomings. More focus is given on trilateral radiolocation especially for indoor human motion tracking. A solution is sought to resolve the problem of algorithm failure with applicability to all areas of positioning and tracking including trilateration when less than three reference points are available.
\end{abstract}

Keyword: Artificial intelligence; Human tracking; Positioning systems; Radiolocation 